\title{
Avaliação dos efeitos de uma intervenção educativa para promoção do uso da Terapia de Reidratação Oral (TRO) em trabalhadores de farmácias
}

\author{
Evaluating the potential of an intervention \\ aimed at promoting oral rehydration therapy \\ (ORT) by educating pharmacy employees
}

Maria de Lourdes Oshiro 1,2

Lia Lusitana Cardozo de Castro 2

1 Secretaria de Estado de Saúde do Mato Grosso do Sul. Av. Senador Filinto Muller 1480, Campo Grande, MS 79074-460, Brasil.

2 Grupo de Pesquisa em Uso Racional de Medicamentos, Universidade Federal do Mato Grosso do Sul. Cidade Universitária, C. P. 549, Campo Grande, MS 79070-900, Brasil.

\begin{abstract}
Diarrhea is a major cause of morbidity and mortality in childhood, and Brazilians rely heavily on pharmacies for the resolution of this and other health problems. To promote the rational use of both pharmaceuticals and oral rehydration therapy (ORT), an intervention study was performed in pharmacies in Southwestern Brazil. Semi-structured interviews showed oral rehydration solution, or ORS (50\%), antidiarrheals (39\%), trimethoprim-sulfamethoxazole (27\%), and yeast (22\%) to be the most frequently suggested drugs, whereas questionnaire responses were ORS (75\%), trimethoprim-sulfamethoxazole (25\%), and yeast (25\%), thus revealing that more than one methodology is needed if reliable data are to be obtained. An educational intervention was applied to $86.7 \%$ of pharmacies, but acquisition of knowledge on management of diarrhea did not prevent pharmacy workers from suggesting antidiarrheal drugs instead of ORS alone. In order to have pharmacy workers comply with official protocols for episodes of diarrhea, interventions should include regulatory measures on drugs that are contraindicated for children, and the role of pharmacies and pharmacists should be reviewed.
\end{abstract}

Key words Oral Rehydration Therapy; Pharmacoepidemiology; Drug Utilization

Resumo A diarréia constitui uma das principais causas de morbi-mortalidade na infância. As farmácias são locais bastante procurados pela população para resolução de problemas de saúde. Visando contribuir para o uso racional de medicamentos, mediante promoção da TRO, foi realizado um estudo de intervenção em farmácias de Corumbá e Ladário. Os medicamentos mais indicados foram SRO (50\%), antidiarréicos (39\%), Sulfametoxazol/Trimetoprim (27\%) e levedo (22\%) na entrevista semi-estruturada; SRO (75\%), Sulfametoxazol/Trimetoprim (25\%) e levedo (25\%) nas respostas ao questionário, demonstrando a importância do uso de mais de uma metodologia para a obtenção de dados fidedignos. A intervenção educativa foi realizada em 86,7\% das farmácias. Os resultados mostraram que mesmo tendo adquirido conhecimento do manejo adequado da diarréia, os trabalhadores de farmácia ainda recomendam outros medicamentos $e$ não SRO exclusivamente. O trabalho evidencia que para conseguir a adesão dos trabalhadores de farmácia aos protocolos oficiais para episódios diarréicos, necessita-se também de medidas normativas em relação aos medicamentos contra-indicados para crianças e reformular as atividades da farmácia e do farmacêutico.

Palavras-chave Terapia de Reidratação Oral; Famacoepidemiologia; Uso de Medicamentos 


\section{Introdução}

A Organização Mundial da Saúde (OMS), em 1995, estimou que aproximadamente $70 \%$ dos 11,6 milhões de mortes anuais de crianças menores de cinco anos, nos países em desenvolvimento, estão atribuídas a cinco afecções: infecções respiratórias agudas, diarréia, causas perinatais, sarampo e malária (WHO, 1998). Na região das Américas, estas cinco enfermidades são responsáveis por aproximadamente 250.000 mortes de crianças menores de cinco anos, e a diarréia corresponde aproximadamente a $20 \%$ das mortes (OPS, 1998).

Em Mato Grosso do Sul, segundo dados da Secretaria do Estado de Saúde (SES-MS, 1998), em 1994 e 1995, as doenças diarréicas constituíram a segunda causa de mortalidade em menores de um ano. A freqüência dos casos de diarréia em Corumbá, município do estudo, calculada com base nas notificações compulsórias, sofreu flutuações que vão de 330 a 1.403/ 100.000 habitantes, no período de 1993 a 1997, para crianças menores de um ano (Oshiro \& Castro, 1998).

A partir de 1982, o Ministério da Saúde do Brasil (MS, 1993), por intermédio das Secretarias Estaduais de Saúde, adotou a terapia de reidratação oral (TRO) como medida de controle das doenças diarréicas e tem sido comprovado que é uma intervenção apropriada e simples no combate à mortalidade de crianças por diarréia e desidratação.

No entanto, uma pesquisa realizada no Nordeste observou que sua utilização foi de $31 \%$ em 1986 e $25,3 \%$ no ano de 1989 , correspondendo a um decréscimo estatisticamente significativo (Galvão et al., 1994). Dados do Fundo das Nações Unidas para a Infância (UNICEF, 1999) apontam que a utilização da TRO no Brasil, no período de 1990 a 1997, foi de $54 \%$.

Um dos aspectos que influenciam no manejo e controle da doença diarréica é o comportamento das pessoas que vivem o problema no dia a dia, pois a conduta adotada na sua resolução, nem sempre é a preconizada pelo sistema oficial, o que poderá determinar o êxito ou o fracasso do programa proposto por este (Obregón, 1996).

Uma alternativa freqüentemente utilizada pela população para recuperar a saúde são as farmácias, que têm sido, ao longo dos anos, o mais acessível dos serviços de saúde, por estarem disponíveis em maior número que as unidades primárias de saúde, clínicas e hospitais. A farmácia torna-se então um estabelecimento, não somente para prestação de serviços na venda de medicamentos, mas também um lo- cal de informação e educação em saúde, exigindo uma comunicação mais eficiente entre profissionais e usuários.

Blanco (1989), na República da Guatemala, encontrou que as "consultas" mais freqüentes nas farmácias referem-se às causas gastrointestinais, representando $36,1 \%$ do total, e o percentual para crianças menores de 5 anos alcança $60,9 \%$. Verificou-se também que $30 \%$ dos trabalhadores das farmácias opinam que é normal uma criança apresentar diarréia. Na Inglaterra, Goodburn et al. (1991) evidenciaram que a diarréia representou $30 \%$ das "consultas" nas farmácias.

No Brasil, Campos et al. (1985) relatam que $82 \%$ dos balconistas indicam predominantemente medicamentos para tratamento sintomático e antibióticos para crianças com diarréia e apenas $18 \%$ recomendam procurar um serviço médico.

Muitas organizações de saúde realizam programas de capacitação para melhorar a maneira que os médicos, enfermeiros e trabalhadores de saúde tratam a diarréia, porém, até pouco tempo, não se prestava nenhuma atenção aos vendedores de medicamentos do setor privado (AHRTAG, 1995).

O farmacêutico é um profissional de saúde bastante indicado para contribuir favoravelmente nos cuidados primários de saúde, pois muitos medicamentos não prescritos são adquiridos em farmácias e dependem de orientações adequadas para seu uso correto (Galloway \& Eby, 1971; WHO, 1994).

É importante salientar que, nem sempre, é o farmacêutico quem atende o usuário na farmácia, muitas vezes é um leigo que pode fornecer orientações inapropriadas e até prejudiciais ao manejo do processo diarréico. Freqüentemente são recomendados medicamentos antidiarréicos ineficazes em lugar da solução de Sais de Reidratação Oral (SRO). As razões deste procedimento incluem falta de conhecimento, disponibilidade de medicamentos inapropriados, demanda dos consumidores, prescrição inadequada pelos médicos, maior margem de lucro com os antidiarréicos do que com a SRO e legislação que não promove o uso racional de medicamentos.

A Política Nacional de Medicamentos define uso racional de medicamentos como o processo que compreende a prescrição apropriada; a disponibilidade oportuna e a preços acessíveis; a dispensação em condições adequadas; e o consumo nas doses indicadas, nos intervalos definidos e no período de tempo indicado de medicamentos eficazes, seguros e de qualidade (MS, 1999). 
Nas últimas décadas, principalmente nos Estados Unidos, Espanha e Argentina, a farmácia vem sofrendo grandes mudanças para alcançar suas metas na atenção à saúde, adaptando-se a uma nova prática farmacêutica denominada Atenção Farmacêutica e com resultados favoráveis à sociedade (Metge et al., 1998; Peretta \& Ciccia, 1998; Reutzel et al., 1999).

Atenção Farmacêutica foi definida por Douglas Hepler e Linda Strand como a dispensação responsável do medicamento com o propósito de alcançar resultados que implementem a qualidade de vida do paciente (Strand et al., 1992).

No Brasil, as farmácias e os farmacêuticos começam a rever suas atividades voltadas ao paciente, para proporcionar orientações seguras e confiáveis, diminuindo os problemas que possam ocorrer com o medicamento. O processo de mudança caminha vagarosamente em relação a outros países, com atividades incipientes de orientação farmacêutica em alguns locais do Brasil (Amorim et al., 1999; Castro et al., 2000).

O objetivo deste trabalho foi avaliar os efeitos de uma intervenção educativa preconizada pela OMS para implementação do uso da TRO, em trabalhadores de farmácias particulares.

\section{Metodologia}

A pesquisa utilizou o método proposto pela OMS compreendendo três etapas com seis estratégias de coleta de dados para conhecer a atuação das farmácias diante de um episódio diarréico, uma intervenção com elaboração de um programa de treinamento no manejo adequado da diarréia em crianças e avaliação destes resultados (WHO, 1993). O fluxograma das etapas realizadas nesta pesquisa encontra-se na Figura 1.

O estudo foi realizado nos municípios de Corumbá e Ladário, por terem apresentado a maior taxa de morbi-mortalidade por diarréia em menores de cinco anos no Estado de Mato Grosso do Sul, no período de julho de 1998 a setembro de 1999. Estabeleceu-se que o trabalhador de farmácia que participasse de qualquer das estratégias seria representativo da conduta da farmácia, não necessitando ser o mesmo em todas as estratégias.

Primeira etapa: visando conhecer as atitudes e práticas do manejo da diarréia aguda, nas farmácias de Corumbá e Ladário, foram utilizados três instrumentos: entrevista semi-estruturada, questionário e grupo-foco.

A entrevista semi-estruturada foi realizada em 18 farmácias/drogarias, que representam o universo da pesquisa conforme dados fornecidos pelo Conselho Regional de Farmácia (CRFMS). Esta entrevista foi aplicada por alunas do curso de pedagogia da Universidade Federal de Mato Grosso do Sul (UFMS) que se apresentavam como "mães" aos trabalhadores das farmácias e pediam orientação para o filho com diarréia.

O segundo instrumento utilizado foi o questionário aplicado ao trabalhador de farmácia abordando os seguintes tópicos: caracteriza-

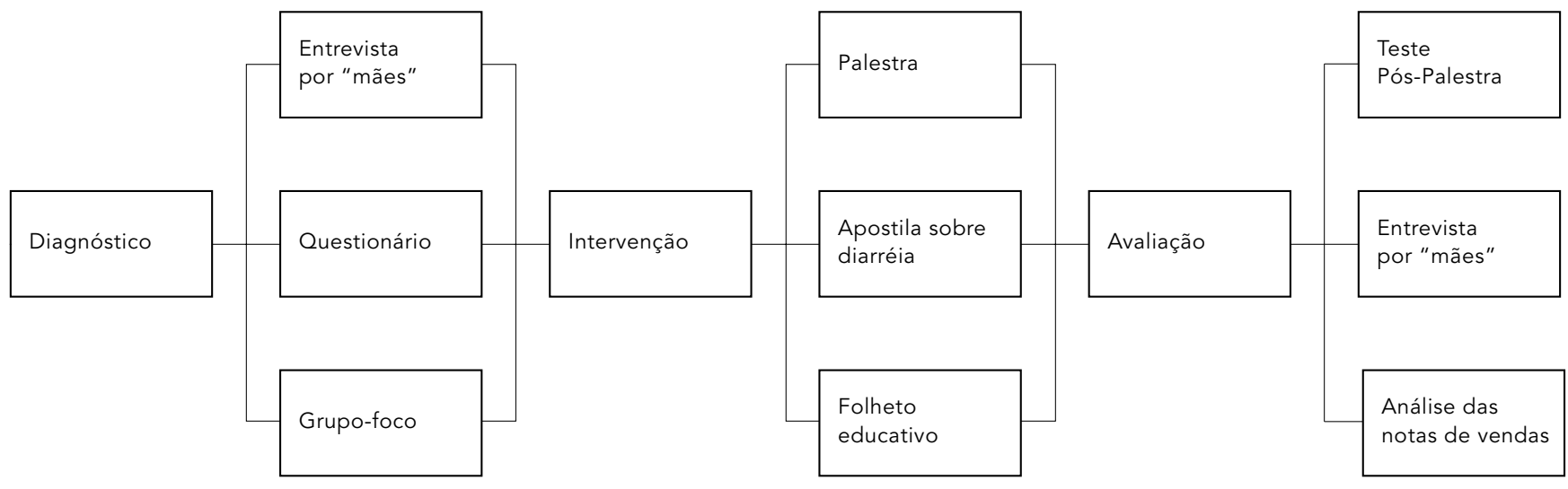


ção da farmácia, conhecimento sobre diarréia e seu tratamento, informações sobre SRO e antidiarréicos e as fontes de informação de medicamentos (WHO, 1993).

O terceiro instrumento utilizado foi o grupo-foco, que é uma entrevista grupal, método de investigação em que se trabalha com coleta de dados de forma coletiva, sendo empregado para conhecer as razões da conduta dos trabalhadores de farmácia na orientação a usuários com episódios diarréicos. Os grupos foram estratificados por sua função na farmácia: farmacêuticos, proprietários leigos e balconistas.

Segunda etapa: aplicação da intervenção educativa, para a qual foram convidados pessoalmente os proprietários de farmácias/drogarias de Corumbá e Ladário, a partir de março de 1999. À medida que os proprietários aceitavam participar do processo educativo, a palestra era realizada no próprio estabelecimento.

A intervenção educativa foi elaborada pelo Grupo de Pesquisa em Uso Racional de Medicamentos (GRUPURAM). Realizou-se uma palestra sobre os problemas e importância do uso adequado de medicamentos, o manejo adequado no tratamento das diarréias agudas em crianças, a TRO e a importância da farmácia na Atenção Primária de Saúde, ministrada por uma das pesquisadoras, sendo fornecida posteriormente uma apostila sobre o assunto.

Ao término da palestra, solicitou-se aos participantes a elaboração de um folheto educativo destinado às mães de crianças com problema diarréico, o qual possibilitaria a cada estabelecimento farmacêutico contribuir ativamente para a melhoria deste problema.

Perante o insucesso dessa estratégia, nas farmácias/drogarias de Corumbá e Ladário, foram utilizados os folhetos educativos da Secretaria de Estado de Saúde "Diarréia e Desidratação" que foram enviados a cada farmácia/drogaria participante, para complementar as orientações dadas aos usuários.

Terceira etapa: após, aproximadamente, cinqüenta dias da última palestra, iniciou-se a avaliação da intervenção. A primeira estratégia de avaliação foi enviar a cada um dos participantes um teste com nove questões semelhantes às do questionário inicial, no que se refere ao conhecimento sobre diarréia, tratamento, SRO e antidiarréicos (Tabela 1).

Quatro meses após a intervenção educativa, foi realizada a segunda avaliação que consistiu de uma entrevista semi-estruturada, aplicada por "mães", semelhante à estratégia inicial, buscando orientação nas farmácias para resolução de um episódio diarréico em criança menor de cinco anos de idade.
Na terceira estratégia realizou-se uma comparação das notas de venda de produtos para diarréia da principal distribuidora do Estado, para os municípios do estudo, no momento anterior (1998) e posterior à intervenção (1999).

\section{Resultados e discussão}

Na entrevista semi-estruturada, verificou-se baixa indicação de SRO e recomendação de medicamentos adicionais ou mesmo substituindo SRO (Tabela 2).

Os resultados obtidos por este instrumento foram semelhantes a outros trabalhos dessa natureza (Campos et al., 1985; Cardoso et al., 1995; Goodburn et al., 1991). As questões mais formuladas pelos trabalhadores de farmácia foram relativas à idade da criança (15), presença de febre e vômitos (11) e duração da diarréia (10).

Quanto à resolução do episódio diarréico, 94,4\% das farmácias indicaram algum medicamento. Apenas $50 \%$ indicaram SRO; todavia, acompanhada de outro medicamento. Os antidiarréicos foram indicados sem SRO em sete casos e os antimicrobianos em cinco casos com ou sem a presença de SRO (Tabela 2).

As informações dadas ao usuário em relação ao medicamento foram insuficientes e realizadas de maneira descompromissada, deixando a mãe decidir o curso do tratamento.

O questionário foi aplicado aos trabalhadores de farmácia nos 16 estabelecimentos que ainda permaneciam em atividade. Verificou-se que mais de $50 \%$ permaneciam abertas em torno de 13 horas e eram de propriedade leiga. Metade das farmácias atendiam de 50 a 150 usuários por dia; $87 \%$ atendiam até cinqüenta usuários sem prescrição médica. Na resolução da doença diarréica, $75 \%$ das farmácias atendiam diariamente até vinte usuários e $69 \%$ atendiam, por dia, em torno de 0 a 5 crianças menores de cinco anos com diarréia, o que correspondia a aproximadamente $2 \%$ do total dos usuários de farmácias dos municípios de Corumbá e Ladário.

A gravidade do problema diarréico é maior em crianças, mas os adultos foram os que mais procuraram as farmácias; dos trezentos usuários com diarréia atendidos nas farmácias do estudo, 49 foram crianças menores de cinco anos, correspondendo a quase $16 \%$ do total dos usuários atendidos com esta patologia.

As 16 farmácias/drogarias do estudo atendiam diariamente em torno de 2.500 usuários por dia, seja comprando medicamentos ou solicitando alguma orientação ao trabalhador de farmácia, terapêutica ou não. Confrontadas 
Algumas questões sobre diarréia e o seu tratamento.

Circule a alternativa certa e em algumas questões pode ter mais de uma alternativa certa.

1) Quais das seguintes causas de diarréia em crianças menores de 5 anos você diria que é o mais comum em Corumbá?

a) comidas condimentadas;

b) água contaminada;

c) desmame:

d) outros, quais

2) Qual a principal razão por que a diarréia é perigosa em crianças menores de 5 anos? a) pode fazê-los perder peso rapidamente;

b) crianças com diarréia são mais susceptíveis de adquirir outras infecções mais rapidamente; c) a diarréia faz perder importantes líquidos e sais do corpo da criança;

d) realmente não é perigosa, mas deixa a criança desconfortável.

3) Quais são os sinais que indicam que um caso de diarréia em crianças menores de 5 anos pode ser causado por bactérias? (Circule todas que se aplicam.)

a) a criança não tem apetite;

b) a evacuação da criança possui sangue;

c) a criança está com febre;

d) evacuações mais do que 5 vezes ao dia.

4) Quando uma criança com diarréia deve ser levada para atendimento médico? (Circule todas que se aplicam.)

a) quando uma diarréia dura mais do que 3 dias:

b) quando a criança chora muito;

c) quando há sangue na evacuação da criança;

d) quando a criança está com febre.

5) Quando uma criança com diarréia não tomaria Solução Reidratante Oral (SRO)?

a) quando sua febre é alta;

b) quando está vomitando muito;

c) quando sua diarréia possui sangue;

d) SRO pode sempre ser tomado.

6) O que SRO faz para a criança com diarréia?

a) SRO elimina alguns dos organismos que causam diarréia no intestino da criança;

b) SRO repõe a água e os sais minerais que foram perdidos com a diarréia;

c) SRO ajuda a diminuir as evacuações;

d) SRO faz todas estas coisas.

7) Quando os antidiarréicos são úteis para uma criança menor de 5 anos com diarréia aguda?

a) somente Quando a diarréia é causada por bactéria;

b) somente Quando tomado junto com SRO;

c) são úteis em todos os tipos de diarréia;

d) nunca são úteis.

8) Quais são os riscos dos medicamentos antidiarréicos? (Circule todas que se aplicam.)

a) desviam o uso de SRO

b) representam despesas desnecessárias;

c) podem causar desidratação;

d) podem ser perigosos.

9) Quando os antibióticos são úteis para uma criança menor de 5 anos com diarréia?

a) somente quando a diarréia é causada por Shigella (fezes com sangue e muco);

b) somente quando usado conjuntamente com SRO;

c) são úteis em todos os tipos de diarréia;

d) nunca são úteis. 
Tabela 2

Orientações fornecidas pelos trabalhadores das farmácias de Corumbá

e Ladário às mães de crianças menores de cinco anos com diarréia.

Julho de 1998 a setembro de 1999.

$\left.\begin{array}{lcr}\hline \text { Orientações } & \text { Farmácia } \mathbf{n}=\mathbf{1 8} & \% \\ \hline \text { SRO+levedo } & 2 & 11,1 \\ \text { SRO+ STZ/TRM com } & 4 & 22,2 \\ \text { ou sem atapulgita } & & \\ \text { SRO+AD } & 3 & 16,7\end{array}\right]$

$\mathrm{SRO}=$ Sais de reidratação oral; levedo $=$ Saccharomyces boulardi-17 ou cerevisae $\mathrm{STZ/TRM} \mathrm{=} \mathrm{Sulfametoxazol/Trimetoprim;} \mathrm{AD} \mathrm{=} \mathrm{Antidiarréico} \mathrm{(furazolidona} \mathrm{e/ou}$ difenoxilato com $1 \%$ de atropina, loperamida)

com as 11 Unidades de Saúde da região, onde o atendimento era em torno de 670 pessoas por dia, este número é muito superior (SES-MS, 1999). Este dado demonstra a importância da farmácia, que é para a população, na maioria das vezes, o único local ao qual recorre para adquirir medicamentos e, por conseqüência, sofre grande influência desta na decisão terapêutica e nos cuidados à saúde.

Mesmo nos países desenvolvidos, como a Suécia, o atendimento nas farmácias é muito maior do que nos centros de atenção primária (Hammarström \& Westerholm, 1988).

Os motivos pelos quais a mãe busca a farmácia estão relacionados com o atendimento das unidades de saúde, seja por estas estarem fechadas à noite, fins de semana e feriados, seja por falta de profissionais de saúde, seja por falta de medicamentos nas unidades sanitárias.

Quanto à função do respondente ao questionário, verificou-se que $50 \%$ eram proprietários, seguidos por balconistas. A formação dos respondentes, com exceção dos farmacêuticos, ocorreu nos próprios locais de trabalho (farmácias/drogarias), por meio do contato direto com os medicamentos ou transmitidos pelo trabalhador mais antigo ou mesmo pela associação de receitas médicas anteriores com sintomas descritos pelo usuário.

O procedimento adequado (OMS, 1994), em grande parte dos casos de diarréia aguda, é o uso exclusivo do reidratante oral que foi recomendado apenas por um balconista. Mais de $80 \%(81,3 \%)$ referiram que recomendavam, além da SRO, outro medicamento (levedo, sulfametoxazol/trimetoprim).
A importância da SRO, ou soro oral, é amplamente divulgada pelos meios de comunicação, no entanto, há uma certa distância entre o seu conhecimento e a sua utilização efetiva pelas mães, que dependerá do valor que é dado por quem o recomenda (Oshiro \& Castro, 1997). Pesquisas realizadas em quatro países asiáticos mostraram que o emprego de medicamentos é muito mais comum que o uso da SRO. A SRO foi empregada de $9 \%$ a $21 \%$ de todos os episódios diarréicos e medicamentos de 22 a $68 \%$. A terapêutica com mais de um medicamento foi freqüente nessa pesquisa (OMS, 1991).

Quanto à alimentação, os trabalhadores de farmácia responderam que recomendavam às mães de criança com diarréia alterar a alimentação, introduzindo frutas como banana, maçã, alimentos sem gordura; aumentar líquidos como água de coco, água de arroz, iogurte e alguns informaram que aconselhavam suspender o leite, exceto o leite materno.

Ao se analisar o questionário, no mês de janeiro de 1999, observou-se que existiam em circulação em torno de seis marcas diferentes de SRO e uma oferta bem maior de outros medicamentos utilizados para a diarréia.

Os produtos à venda para diarréia foram classificados segundo Capella \& Laporte (1993), verificando-se a existência de 13 medicamentos para uso infantil com valor intrínseco elevado, dois de valor intrínseco relativo, cinco de valor intrínseco duvidoso ou nulo e seis de valor intrínseco inaceitável. Dos medicamentos à venda somente dois constavam na Lista de Medicamentos Essenciais da OMS e na Relação Nacional de Medicamentos Essenciais - RENAME (Sulfametoxazol/Trimetoprim e Sais de Reidratação Oral OMS).

As fontes de informação sobre medicamentos relatadas foram a bula dos medicamentos e o Dicionário de Especialidades Farmacêuticas (DEF). Também foram citados livros de referência (farmacologia, patologia e terapêutica). Os respondentes consideraram ainda que a fonte mais importante é o DEF, seguida pelos livros e boletins da área, bem como as prescrições médicas. A fonte de informação consultada era voltada para a venda do medicamento (indicações e vantagens) e não para a orientação ao paciente, isenta e independente da indústria farmacêutica.

Verificou-se que quando o farmacêutico não é o proprietário da farmácia, a dedicação aos serviços farmacêuticos e atenção ao paciente deixa muito a desejar. Os resultados do grupo-foco mostraram que os farmacêuticos estavam atualizados com os protocolos oficiais para o tratamento correto da diarréia aguda, 
enquanto os balconistas tinham conhecimento do reidratante oral, mas não como a principal alternativa na diarréia ou essencial na reposição hidroeletrolítica de diarréia de qualquer etiologia. Nesta estratégia, a adesão ao convite foi muito pequena, havendo apenas uma reunião com os farmacêuticos e uma com os balconistas.

Comparando os instrumentos utilizados para conhecer como os trabalhadores de farmácia forneciam orientações, verificou-se que na entrevista, $44 \%$ recomendavam somente medicamentos sem SRO e a SRO foi aconselhada em $50 \%$ dos casos (Tabela 2) e, no questionário, $81 \%$ das recomendações incluíam o tratamento com SRO. As respostas obtidas pelo questionário foram mais coerentes com as recomendações dos protocolos e guias oficiais. Na entrevista, os trabalhadores de farmácia mostraram-se mais inadequados quanto às orientações medicamentosas para diarréia, o que é preocupante, visto que este comportamento ocorreu diante de uma situação supostamente real.

No Brasil, cada vez mais, o uso indiscriminado e desnecessário de medicamentos vem se tornando um problema de saúde pública. A maioria dos serviços farmacêuticos oferecidos nas farmácias e drogarias particulares é executado por trabalhadores sem nenhuma formação específica e apropriada para dispensar medicamentos ou fornecer conselhos sobre a terapia medicamentosa.

Em 1997, ocorreu na Tailândia a Primeira Conferência Internacional sobre a Melhoria do Uso de Medicamentos (CIMUM), que evidenciou que intervenções formativas bem preparadas, em grupos pequenos ou grandes, podem melhorar os resultados da prescrição em torno de $15 \%$. O efeito da formação parece aumentar nas intervenções que utilizam uma mistura de métodos formativos (palestras, representação de situações, solução de problemas) que enfocam situações concretas, compreendem a formação no local de trabalho e utilizam como formadores os líderes de opinião ou pessoal do distrito (OMS, 1997).

Na atenção primária de saúde observa-se que a simples difusão de informação não é suficiente para obter uma melhora mensurável no comportamento. Os pacientes ou usuários de medicamentos necessitam de informações compreensíveis acerca dos medicamentos que tomam, por que tomar e como tomar. Para isso, os prescritores e dispensadores precisam de formação para melhorar sua comunicação com os pacientes.

Neste trabalho, a atividade de intervenção educativa para controlar a diarréia objetivou a mudança da prática dos trabalhadores em farmácias, estimulando-os a priorizar a TRO como base principal do tratamento, conseqüentemente, contribuindo para modificar as atitudes errôneas da população em relação à doença.

Dos 18 estabelecimentos farmacêuticos cadastrados no CRF-MS nas cidades de Corumbá e Ladário em julho de 1998, quatro encerraram suas atividades neste período, dois recusaramse a participar e um iniciou suas atividades e foi incluído na pesquisa. Participaram da intervenção educativa 13 farmácias, correspondendo a $86,7 \%$ do total.

A maioria dos proprietários das farmácias / drogarias aceitou bem a intervenção educativa, uma vez que não havia necessidade de deslocamento e, assim, os seus funcionários permaneceriam no próprio estabelecimento.

Aderiram à intervenção educativa 34 trabalhadores de farmácia que estavam permanentemente em contato com o usuário, correspondendo a um percentual de $35,8 \%$, porém participaram do processo educativo mais dez pessoas com outras atividades na farmácia, que eventualmente atendiam a clientela. Verificouse na pesquisa que $81,2 \%$ da clientela que foi treinada era composta por proprietários leigos e balconistas.

Solicitou-se a elaboração de um folheto educativo relacionado ao problema, mas apenas dois estabelecimentos realizaram esboços do folheto educativo revelando que, em atividade passiva como a palestra, os trabalhadores cooperam; contudo, quando se exige esforço ativo de cada um, estes se mostram resistentes. Um dado preocupante constatado nesta pesquisa foi a grande ausência do profissional farmacêutico, o qual deveria estar presente e participativo nos cuidados de atenção à saúde da população.

Na etapa de avaliação, a primeira estratégia utilizada foi um teste com nove questões (Tabela 1), semelhantes ao questionário inicial (conhecimento), enviado aos trabalhadores das 13 farmácias que participaram da intervenção educativa. Retornaram 41 testes (95,3\%), que foram analisados por farmácia, para tal foi calculada a média do conhecimento dos trabalhadores de cada uma delas e confrontou-se a mesma com o conhecimento anterior obtido pelo questionário.

A Tabela 3 apresenta a comparação do nível de conhecimento pré e pós-palestra das farmácias participantes do processo educativo. $\mathrm{O}$ intervalo de tempo foi importante para verificar se houve assimilação do conteúdo transmitido. Os resultados pós-palestra mostraram-se estatisticamente superiores ao conhecimento de- 
Tabela 3

Nível de conhecimento dos trabalhadores das farmácias/drogarias de Corumbá e Ladário no pré e pós-processo educativo. Julho de 1998 a setembro de 1999.

\begin{tabular}{lccr}
\hline Depois & $\begin{array}{l}\text { Antes } \\
\text { suficiente }\end{array}$ & $\begin{array}{l}\text { Conhecimento } \\
\text { insuficiente }\end{array}$ & Total \\
\hline Conhecimento suficiente & 3 & 7 & 10 \\
Conhecimento insuficiente & 2 & 0 & 2 \\
Total & 5 & 7 & 12
\end{tabular}

Utilizando-se a prova binomial obteve-se um nível descritivo de 0,125.

Logo, no nível de significância de $5 \%$, afirmamos que houve diferença

entre os resultados da pré e da pós-intervenção educativa. monstrado no questionário, conforme mostra a prova binomial realizada, indicando que uma intervenção educativa reformula e acrescenta novos conteúdos na formação do trabalhador em farmácia, principalmente do pessoal leigo.

Por sua importância, a resposta 1 do teste pós-intervenção (Tabela 1) é apresentada separadamente na Tabela 4 . A causa mais comum de diarréia em criança, na opinião dos participantes, é a água contaminada (71\%), sugerindo uma diarréia infecciosa, generalizando a indicação do antimicrobiano, inapropriadamente. Outra causa bastante citada foi a alimentação condimentada (32\%), talvez relacionada aos hábitos alimentares da Bolívia, país que faz divisa com o município. A patologia também foi associada a baixas condições sócio-econômicas, salientando condições precárias de vida, a falta de higiene, falta de saneamento básico (34\%) e, também, ao desmame precoce (12\%).

Outra questão polêmica foi a de número 7, quanto à utilização de antidiarréicos; grande parte dos trabalhadores de farmácia não aceita que estes medicamentos sejam ineficazes, principalmente nas farmácias de proprietários leigos.

Deve-se considerar que há controvérsias quanto à adesão aos protocolos elaborados pela OMS/UNICEF para o manejo adequado de diarréia, mesmo por parte dos médicos. Em estudos citados por Paredes et al. (1996), como a pesquisa de Muninjaya e colaboradores, verificou-se que $68 \%$ das mães relataram dar TRO para suas crianças com diarréia, porém, a metade delas também dava medicamentos para suas crianças e $90 \%$ destes eram prescritos por médicos; o trabalho de Gani e colaboradores, na Indonésia, demonstrou a discrepância entre conhecimento e prática dos médicos no tratamento da diarréia em que, em $54 \%$ dos casos observados, foram prescritos antimicrobianos e a SRO era prescrita menos freqüentemente do que o reportado. Paredes et al. (1996) constataram no Paquistão que os antidiarréicos foram prescritos por $60 \%$ de clínicos gerais e $29 \%$ dos pediatras e os antibacterianos foram prescritos em $66 \%$ dos clínicos gerais e $50 \%$ dos pediatras.

Mesmo nos países desenvolvidos, como os Estados Unidos, a prática médica não é homogênea, como foi observado em pesquisa realizada com pediatras comunitários e coordenadores de departamentos de pediatria, verificando que as atitudes e práticas relacionadas à terapia líquida oral e alimentação durante a diarréia aguda, divergem das recomendações dos protocolos oficiais americanos (Snyder, 1991).

A literatura técnico-científica é também contraditória em relação às recomendações da OMS quanto à utilização de medicamentos no tratamento da diarréia. O sistema informatizado Micromedex (1999) relata que, embora a produção e venda de produtos na forma líquida de loperamida tenha sido suspensa nos países em desenvolvimento, a produção e comercialização deste fármaco não sofreu restrições nos Estados Unidos onde a Food and Drug Administration (FDA) aprovou em 1990 seu uso em crianças acima de dois anos de idade. Difenoxilato é outro antidiarréico com atividade opióide, também liberado pelo FDA para crianças acima de dois anos de idade. No Brasil, por intermédio da Portaria 344, o difenoxilato está com a venda controlada e sujeita à retenção da receita médica (Brasil, 1999).

Estes fatos mostram que há outros fatores atuantes, além do conhecimento específico da doença, e que somente intervenções educativas isoladas não são suficientes para transformar uma prática inadequada e, ao mesmo tempo, muito difundida, sendo necessárias outras estratégias para alcançar este objetivo.

Na Tabela 5, comparam-se os resultados da entrevista quanto à indicação da SRO antes e após a intervenção educativa. Neste momento os dados foram coletados de apenas 13 farmácias, pois duas não estavam mais em funcionamento e três se recusaram a participar da intervenção educativa. Analisando os resultados obtidos nos dois momentos de coleta de dados, verificou-se que houve um aumento estatisticamente significativo de indicação de SRO, conforme está demonstrado pela prova binomial realizada. Entretanto, permanece a recomendação de outros medicamentos com ou sem SRO em 92,3\%, nas duas situações.

Ainda que os trabalhadores de farmácias/ drogarias tenham conhecimento da forma 
adequada de tratamento da diarréia aguda em crianças, demonstrado pelo teste aplicado (Tabela 3), o fato da farmácia ser um estabelecimento comercial contribuiu para uma indicação irracional e inadequada na prática cotidiana. A subsistência da farmácia particular é obtida pela venda de medicamentos e correlatos, tendo como poderoso aliado deste comércio a indústria farmacêutica que estimula os prescritores, dispensadores e a população com suas sofisticadas e atraentes propagandas a utilizarem, cada vez mais, medicamentos.

Constatou-se pelos dados da pesquisa que, mesmo tendo conhecimento da importância da reposição de água e eletrólitos, os trabalhadores de farmácia mantêm a indicação de medicamentos para interromper a diarréia à base de caulim/pectina e levedo, que são contra-indicados por não terem comprovação científica de sua eficácia. Verificou-se também a utilização de Sulfametoxazol/Trimetoprim, e o quadro exposto na entrevista semi-estruturada não justificava a recomendação deste produto em 4 e 5 indicações respectivamente, anteriores e posteriores ao processo educativo. Com a Portaria 344/98 (Brasil, 1999), que entrou em vigor em 01/02/1999, houve um impacto positivo reduzindo a indicação de medicamentos antimotílicos, de 6 para 1 .

A comparação das notas de venda de produtos para diarréia, apontou que ocorreu diminuição na comercialização de produtos para diarréia, de 24.066 unidades (1998) para 16.365 unidades (1999). A diminuição da venda de produtos à base de difenoxilato foi mais acentuada que das demais especialidades, (4.670 para 1.210 unidades), em virtude de restrições feitas pelo Ministério da Saúde à comercialização destes medicamentos, o que prova a necessidade de uma legislação eficaz para racionalizar o uso de medicamentos.

\section{Considerações finais}

O controle das doenças diarréicas agudas, principalmente em crianças, requer que todos os profissionais de saúde envolvidos tenham conhecimento do manejo adequado do seu tratamento; portanto, não somente os profissionais do setor público mas também do setor privado.

Demonstrou-se que as farmácias e drogarias podem se tornar postos avançados de saúde quando bem utilizadas, devido ao grande número de usuários a que atendem, ao fácil acesso e à sua disponibilidade. Nos locais de estudo, as farmácias representam $48,5 \%$ de todos os estabelecimentos envolvidos com saúde
Tabela 4

Opinião dos respondentes sobre as causas mais comuns de diarréia em crianças menores de 5 anos em Corumbá e Ladário ( $n=41$, havendo mais de uma resposta). Julho de 1998 a setembro de 1999.

\begin{tabular}{lcc}
\hline Causas & Respondentes & $\%$ \\
\hline Água contaminada & 29 & 71 \\
Comidas condimentadas & 13 & 32 \\
Falta de higiene & 9 & 22 \\
Desmame precoce & 5 & 12 \\
Falta de saneamento básico & 4 & 10 \\
Desnutrição (alimentação inadequada) & 4 & 10 \\
Virose & 3 & 7 \\
Falta de orientação sanitária & 1 & 2 \\
Condições precárias de vida & 1 & 2
\end{tabular}

Tabela 5

Dados comparativos da prática dos trabalhadores de farmácias/drogarias, em relação aos SRO, anteriores e posteriores à intervenção educativa ( $n=13$ ). Julho de 1998 a setembro de 1999.

\begin{tabular}{lccc}
\hline Depois & SRO não & $\begin{array}{c}\text { Antes } \\
\text { SRO sim }\end{array}$ & Total \\
\hline SRO não & 3 & 4 & 7 \\
SRO sim & 5 & 1 & 6 \\
Total & 8 & 5 & 13
\end{tabular}

Utilizando-se a prova binomial, obteve-se um nível descritivo de 0,3125.

Logo, no nível de significância de $5 \%$, afirmamos que houve diferença na probabilidade de se usar o SRO na pré e na pós-intervenção educativa. $\mathrm{SRO}=$ Sais de reidratação oral

da população como hospitais, clínicas particulares e unidades públicas de saúde.

Os resultados obtidos revelaram que a prática dos trabalhadores de farmácia, frente a um episódio diarréico em crianças menores de cinco anos, é a indicação de medicamentos com ou sem SRO, verificando-se que ainda permanecem condutas consideradas inadequadas $\mathrm{e}$ divergentes dos protocolos oficiais. A intervenção educativa mostrou-se insuficiente, pois a mudança de comportamento nos trabalhadores de farmácia implica, além do conhecimento, outros fatores, principalmente por ser a farmácia particular, no Brasil, um estabelecimento comercial. O uso racional de medicamentos no tratamento da diarréia exige que toda a equipe de saúde esteja integrada e que se cumpram as medidas que regulam a venda e o uso de 
medicamentos, como a Portaria 344/98 (Brasil, 1999). Para melhorar a qualidade do serviço prestado pela farmácia, é fundamental que o profissional farmacêutico contribua, exercendo seu papel de educador da equipe sob sua responsabilidade técnica.

Os resultados deste estudo mostraram que é extremamente necessário reformular a farmácia particular, resgatando o valor desta como equipamento de saúde efetivo na promoção e recuperação da saúde da população.

\section{Referências}

AHRTAG (Appropriate Health Resources \& Technologies Action Group), 1995. Diálogo sobre la Diarrea, 53. Washington, DC: Organización Panamericana de la Salud/Organización Mundial de la Salud.

AMORIM, E. L. C.; LYRA Jr., D. P. \& OLIVEIRA, M. A. C., 1999. Aconselhamento ao paciente: Uma proposta de inclusão no currículo farmacêutico. Pharmacia Brasileira, 15:47-49.

BLANCO, R. R. A., 1989. Conceptos de empleados de farmacia acerca del manejo de la diarrea en niños. Boletín Médico del Hospital Infantil de México, 46:463-468.

BRASIL, 1999. Portaria no 344,12 de maio de 1998: Aprova o regulamento técnico sobre substâncias e medicamentos sujeitos a controle especial. Brasília: Diário Oficial da União, no 21, pp. 29-71, 1 fev. Seção I.

CAMPOS, J. A.; OLIVEIRA, J. S.; COSTA, D. M.; MACHADO, C. D.; ALVARENGA, J. R.; TORRES, L. O.; FERREIRA, M. T.; LADEIRA, R. M. \& CANÇADO, R. L., 1985. Prescrição de medicamentos por balconistas de 72 farmácias de Belo Horizonte/ MG, em maio de 1983. Jornal de Pediatria, 59: 307-312.

CAPELLA, D. \& LAPORTE, J. R., 1993. Métodos aplicados en estudios descriptivos de utilización de medicamentos. In: Principios de Epidemiología del Medicamento (J. R. Laporte \& G. Tognoni, org.), pp. 67-93, Barcelona: Ediciones Científicas y Técnicas.

CARDOSO, A. C.; MIGUÉNS, C.; OLIVEIRA, E.; CARVALHO, L.; SILVA, M. E. \& LEMOS, L., 1995. Diarréia aguda do lactente - $\mathrm{O}$ papel dos profissionais de farmácia na sua orientação. Farmácia Portuguesa, 95:49-52.

CASTRO, L. L. C.; DARVENE, L. S. \& SILVA, E. V., 2000. Relato de uma experiência para viabilizar a prática da orientação farmacêutica nas farmácias comunitárias do Distrito Federal - Brasil. In: IX Congresso da Organização de Farmacêuticos Ibero-Latinoamericanos, Resumos, p. 44, Salvador: Organização de Farmacêuticos Ibero-Latinoamericanos/Universidade Federal da Bahia.

\section{Agradecimento}

Os autores agradecem à pesquisadora Raquel Cymrot do Grupo de Pesquisa em Uso Racional de Medicamentos (GRUPURAM) a análise estatística apresentada neste artigo.

GALLOWAY, S. P. \& EBY, C. E., 1971. Poverty area residents look at pharmacy services. American Journal Pharmacy Hospital, 61:2211-2222.

GALVÃO, C. E. S.; SILVA, A. A. M.; SILVA, R. A.; REIS FILHO, S. A.; NOVOCHADLO, M. A. S. \& CAMPOS, G. J. V., 1994. Terapia de reidratação oral para diarréia aguda em região do nordeste do Brasil, 1986 - 1989. Revista de Saúde Pública, 28:416-422.

GOODBURN, E.; MATTOSINHO, S.; MONGI, P. \& WATERSTON, T., 1991. Management of childhood diarrhoea by pharmacists and parents: Is Britain lagging behind the Third World? BMJ, 302:440443

HAMMARSTRÖN, B. \& WESTERHOLM, B., 1988. Los farmacéuticos al primer plano. Foro Mundial de la Salud, 9:528-532

METGE, C. J.; HENDRICKSEN, C. \& MAINE, L., 1998. Consumer atitudes, behaviors and perceptions about pharmacies, pharmacists and pharmaceutical care. Journal of the American Pharmaceutical Association, 38:37-47.

MICROMEDEX, 1999. Health Care Series. Edition 102. Englewood: Micromedex Incorporation.

MS (Ministério da Saúde), 1993. Assistência e Controle das Doenças Diarréicas. Brasília: MS.

MS (Ministério da Saúde), 1999. Política Nacional de Medicamentos. Brasília: MS

OBREGÓN, P. L., 1996. Acesso ao Conhecimento e Práticas em Relação à Doença Diarréica Aguda na Infância - Um Estudo junto às Mães, Balconistas de Farmácias e Médicos, no Subdistrito de Paz do Sacomã - São Paulo. Dissertação de Mestrado, São Paulo: Faculdade de Saúde Pública, Universidade de São Paulo.

OMS (Organización Mundial de la Salud), 1991. El Manejo Racional de la Diarrea en los Niños. Boletín de Medicamentos Esenciales 11. Geneva: OMS.

OMS (Organización Mundial de la Salud), 1994. Manejo y Prevención de la Diarrea. Pautas Prácticas. Geneva: OMS.

OMS (Organización Mundial de la Salud), 1997. Conferencia Internacional sobre la Mejora del Uso de 
Medicamentos. Boletín de Medicamentos Esenciales 23. Geneva: OMS.

OPS (Organización Panamericana de la Salud), 1998. Atención integrada a las enfermedades prevalentes de la infancia (AIEPI) en las Américas. Boletín Epidemiológico, 19:1-9.

OSHIRO, M. L. \& CASTRO, L. L. C., 1997. Conhecimento de grau de informação das mães de crianças de 0 a 4 anos, sobre Terapia de Reidratação Oral (TRO). In: IV Congresso Brasileiro de Vigilância de Medicamentos, Resumos, p. 114, Curitiba: Sociedade Brasileira de Vigilância de Medicamentos.

OSHIRO, M. L. \& CASTRO, L. L. C., 1998. Série histórica de óbitos por doenças diarréicas em Mato Grosso do Sul. 1990-1996. In: IV Congresso Brasileiro de Epidemiologia, Resumos, p. 350, Rio de Janeiro: ABRASCO.

PAREDES, P.; DE LA PEÑA, M.; GUERRA, E. F; DIAZ, J. \& TROSTLE, J., 1996. Factors influencing physicians prescribing behaviour in the treatment of childhood diarrhoea: Knowledge may not be the clue. Social Science and Medicine, 42:1141-1153.

PERETTA, M. \& CICCIA, G., 1998. Reingeniería de la Práctica Farmacéutica. Buenos Aires: Editorial Médica Panamericana.

REUTZEL, T. J.; DEFALCO, P. G.; HOGAN, M. \& KAZEROONI, P. V., 1999. Evaluation of a pharmaceutical care education series for chain pharmacists using the focus group method. Journal of the American Pharmaceutical Association, 39:226-234.
SES-MS (Secretaria de Estado de Saúde do Mato Grosso do Sul), 1998. Mortalidade Proporcional por Causas mais Freqüentes em Menores de 01 Ano. Campo Grande: SES-MS.

SES-MS (Secretaria de Estado de Saúde do Mato Grosso do Sul). 1999. Relatório de Atendimento nas Unidades de Saúde de Corumbá. Campo Grande: SES-MS.

SNYDER, J. D., 1991. Use and misuse of oral therapy for diarrhea: Comparison of US practices with American academy of pediatrics recomendations. Pediatrics, 87:28-33.

STRAND, L. M.; CIPOLLE, R. J. \& MORLEY, P. C., 1992. Pharmaceutical Care: An Introduction. Kalamazoo: Upjohn Company.

UNICEF (Fundo das Nações Unidas para a Infância), 1999. Situação Mundial da Infância. Brasília: UNICEF.

WHO (World Health Organization), 1993. Diarrhoeal Disease Control Programme Guide for Improving Diarrhoea Treatment Practices of Pharmacies and Licensed Drug Sellers. Geneva: Control Diarrhoeal Disease, WHO

WHO (World Health Organization), 1994. The Role of the Pharmacist in the Health Care System. New Delhi, India, 1988 e Tokyo, Japan, 1993. Report 94/569. Geneva: Pharmaceutical, WHO.

WHO (World Health Organization), 1998. The World Health Report 1998. Life in the $21^{\text {st }}$ Century. A Vision for All. Geneva: WHO.

Recebido em 1 de setembro de 2000

Versão final reapresentada em 26 de junho de 2001

Aprovado em 7 de outubro de 2001 